\title{
Sentence Meta-Embeddings for Unsupervised Semantic Textual Similarity
}

\author{
Nina Poerner* ${ }^{* \dagger}$ and Ulli Waltinger ${ }^{\dagger}$ and Hinrich Schütze* \\ ${ }^{*}$ Center for Information and Language Processing, LMU Munich, Germany \\ ${ }^{\dagger}$ Corporate Technology Machine Intelligence (MIC-DE), Siemens AG Munich, Germany \\ poerner@cis.uni-muenchen.de | inquiries@cislmu.org
}

\begin{abstract}
We address the task of unsupervised Semantic Textual Similarity (STS) by ensembling diverse pre-trained sentence encoders into sentence meta-embeddings. We apply, extend and evaluate different meta-embedding methods from the word embedding literature at the sentence level, including dimensionality reduction (Yin and Schütze, 2016), generalized Canonical Correlation Analysis (Rastogi et al., 2015) and cross-view auto-encoders (Bollegala and Bao, 2018). Our sentence metaembeddings set a new unsupervised State of The Art (SoTA) on the STS Benchmark and on the STS12-STS16 datasets, with gains of between $3.7 \%$ and $6.4 \%$ Pearson's $r$ over singlesource systems.
\end{abstract}

\section{Introduction}

Word meta-embeddings have been shown to exceed single-source word embeddings on word-level semantic benchmarks (Yin and Schütze, 2016; Bollegala and Bao, 2018). Presumably, this is because they combine the complementary strengths of their components.

There has been recent interest in pre-trained "universal" sentence encoders, i.e., functions that encode diverse semantic features of sentences into fixed-size vectors (Conneau et al., 2017). Since these sentence encoders differ in terms of their architecture and training data, we hypothesize that their strengths are also complementary and that they can benefit from meta-embeddings.

To test this hypothesis, we adapt different metaembedding methods from the word embedding literature. These include dimensionality reduction (Yin and Schütze, 2016), cross-view autoencoders (Bollegala and Bao, 2018) and Generalized Canonical Correlation Analysis (GCCA) (Rastogi et al., 2015). The latter method was also used by Poerner and Schütze (2019) for domain-specific Duplicate Question Detection.

Our sentence encoder ensemble includes three models from the recent literature: Sentence-BERT (Reimers and Gurevych, 2019), the Universal Sentence Encoder (Cer et al., 2017) and averaged ParaNMT vectors (Wieting and Gimpel, 2018). Our meta-embeddings outperform every one of their constituent single-source embeddings on STS12-16 (Agirre et al., 2016) and on the STS Benchmark (Cer et al., 2017). Crucially, since our meta-embeddings are agnostic to the contents of their ensemble, future improvements may be possible by adding new encoders.

\section{Related work}

\subsection{Word meta-embeddings}

Word embeddings are functions that map word types to vectors. They are typically trained on unlabeled corpora and capture word semantics (e.g., Mikolov et al. (2013); Pennington et al. (2014)).

Word meta-embeddings combine ensembles of word embeddings by various operations: Yin and Schütze (2016) use concatenation, SVD and linear projection, Coates and Bollegala (2018) show that averaging word embeddings has properties similar to concatenation. Rastogi et al. (2015) apply generalized canonical correlation analysis (GCCA) to an ensemble of word vectors. Bollegala and Bao (2018) learn word meta-embeddings using autoencoder architectures. Neill and Bollegala (2018) evaluate different loss functions for autoencoder word meta-embeddings, while Bollegala et al. (2018) explore locally linear mappings.

\subsection{Sentence embeddings}

Sentence embeddings are methods that produce one vector per sentence. They can be grouped into two categories: 


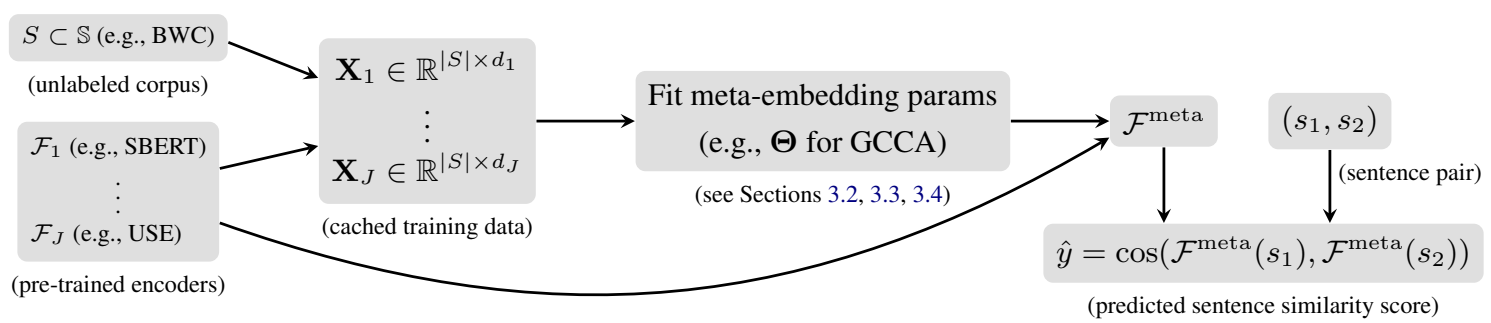

Figure 1: Schematic depiction: Trainable sentence meta-embeddings for unsupervised STS.

(a) Word embedding average sentence encoders take a (potentially weighted) average of pre-trained word embeddings. Despite their inability to understand word order, they are surprisingly effective on sentence similarity tasks (Arora et al., 2017; Wieting and Gimpel, 2018; Ethayarajh, 2018)

(b) Complex contextualized sentence encoders, such as Long Short Term Memory Networks (LSTM) (Hochreiter and Schmidhuber, 1997) or Transformers (Vaswani et al., 2017). Contextualized encoders can be pre-trained as unsupervised language models (Peters et al., 2018; Devlin et al., 2019), but they are usually improved on supervised transfer tasks such as Natural Language Inference (Bowman et al., 2015).

\subsection{Sentence meta-embeddings}

Sentence meta-embeddings have been explored less frequently than their word-level counterparts. Kiela et al. (2018) create meta-embeddings by training an LSTM sentence encoder on top of a set of dynamically combined word embeddings. Since this approach requires labeled data, it is not applicable to unsupervised STS.

Tang and de Sa (2019) train a Recurrent Neural Network (RNN) and a word embedding average encoder jointly on a large corpus to predict similar representations for neighboring sentences. Their approach trains both encoders from scratch, i.e., it cannot be used to combine existing encoders.

Poerner and Schütze (2019) propose a GCCAbased multi-view sentence encoder that combines domain-specific and generic sentence embeddings for unsupervised Duplicate Question Detection. In this paper, we extend their approach by exploring a wider range of meta-embedding methods and an ensemble that is more suited to STS.

\subsection{Semantic Textual Similarity (STS)}

Semantic Textual Similarity (STS) is the task of rating the similarity of two natural language sentences on a real-valued scale. Related applications are semantic search, duplicate detection and sentence clustering.

Supervised SoTA systems for STS typically apply cross-sentence attention (Devlin et al., 2019; Raffel et al., 2019). This means that they do not scale well to many real-world tasks. Supervised "siamese" models (Reimers and Gurevych, 2019) on the other hand, while not competitive with crosssentence attention, can cache sentence embeddings independently of one another. For instance, to calculate the pairwise similarities of $N$ sentences, a cross-sentence attention system must calculate $O\left(N^{2}\right)$ slow sentence pair embeddings, while the siamese model calculates $O(N)$ slow sentence embeddings and $O\left(N^{2}\right)$ fast vector similarities.

Our meta-embeddings are also cacheable (and hence scalable), but they do not require supervision.

\section{Sentence meta-embedding methods}

Below, we assume access to an ensemble of pretrained sentence encoders, denoted $\mathcal{F}_{1} \ldots \mathcal{F}_{J}$. Every $\mathcal{F}_{j}$ maps from the (infinite) set of possible sentences $\mathbb{S}$ to a fixed-size $d_{j}$-dimensional vector.

Word meta-embeddings are usually learned from a finite vocabulary of word types (Yin and Schütze, 2016). Sentence embeddings lack such a "vocabulary", as they can encode any member of $\mathbb{S}$. Therefore, we train on a sample $S \subset \mathbb{S}$, i.e., on a corpus of unlabeled sentences.

\subsection{Naive meta-embeddings}

We create naive sentence meta-embeddings by concatenating (Yin and Schütze, 2016) or averaging ${ }^{1}$ (Coates and Bollegala, 2018) sentence embeddings.

$$
\mathcal{F}^{\text {conc }}\left(s^{\prime}\right)=\left[\begin{array}{c}
\hat{\mathcal{F}}_{1}\left(s^{\prime}\right) \\
\ldots \\
\hat{\mathcal{F}}_{J}\left(s^{\prime}\right)
\end{array}\right]
$$

\footnotetext{
${ }^{1}$ If embeddings have different dimensionalities, we pad the shorter ones with zeros.
} 


$$
\mathcal{F}^{\mathrm{avg}}\left(s^{\prime}\right)=\sum_{j} \frac{\hat{\mathcal{F}}_{j}\left(s^{\prime}\right)}{J}
$$

Note that we length-normalize all embeddings to ensure equal weighting:

$$
\hat{\mathcal{F}}_{j}(s)=\frac{\mathcal{F}_{j}(s)}{\left\|\mathcal{F}_{j}(s)\right\|_{2}}
$$

\subsection{SVD}

Yin and Schütze (2016) use Singular Value Decomposition (SVD) to compactify concatenated word embeddings. The method is straightforward to extend to sentence meta-embeddings. Let $\mathbf{X}^{\text {conc }} \in \mathbb{R}^{|S| \times \sum_{j} d_{j}}$ with

$$
\mathbf{x}_{n}^{\text {conc }}=\mathcal{F}^{\text {conc }}\left(s_{n}\right)-\mathbb{E}_{s \in S}\left[\mathcal{F}^{\text {conc }}(s)\right]
$$

Let $\mathbf{U S V}^{T} \approx \mathbf{X}^{\text {conc }}$ be the $d$-truncated SVD. The SVD meta-embedding of a new sentence $s^{\prime}$ is:

$$
\mathcal{F}^{\mathrm{svd}}\left(s^{\prime}\right)=\mathbf{V}^{T}\left(\mathcal{F}^{\mathrm{conc}}\left(s^{\prime}\right)-\mathbb{E}_{s \in S}\left[\mathcal{F}^{\text {conc }}(s)\right]\right)
$$

\subsection{GCCA}

Given random vectors $\mathbf{x}_{1}, \mathbf{x}_{2}$, Canonical Correlation Analysis (CCA) finds linear projections such that $\boldsymbol{\theta}_{1}^{T} \mathbf{x}_{1}$ and $\boldsymbol{\theta}_{2}^{T} \mathbf{x}_{2}$ are maximally correlated. Generalized CCA (GCCA) extends CCA to three or more random vectors. Bach and Jordan (2002) show that a variant of GCCA reduces to a generalized eigenvalue problem on block matrices:

$$
\begin{array}{r}
\rho\left[\begin{array}{ccc}
\boldsymbol{\Sigma}_{1,1} & \mathbf{0} & \mathbf{0} \\
\mathbf{0} & \boldsymbol{\Sigma}_{\ldots} & \mathbf{0} \\
\mathbf{0} & \mathbf{0} & \boldsymbol{\Sigma}_{J, J}
\end{array}\right]\left[\begin{array}{l}
\boldsymbol{\theta}_{1} \\
\ldots \\
\boldsymbol{\theta}_{J}
\end{array}\right] \\
=\left[\begin{array}{ccc}
\mathbf{0} & \boldsymbol{\Sigma} & \boldsymbol{\Sigma}_{1, J} \\
\boldsymbol{\Sigma}_{\ldots} & \mathbf{0} & \boldsymbol{\Sigma}_{\ldots} \\
\boldsymbol{\Sigma}_{J, 1} & \boldsymbol{\Sigma}_{\ldots} & \mathbf{0}
\end{array}\right]\left[\begin{array}{l}
\boldsymbol{\theta}_{1} \\
\ldots \\
\boldsymbol{\theta}_{J}
\end{array}\right]
\end{array}
$$

where

$$
\begin{aligned}
\boldsymbol{\Sigma}_{j, j^{\prime}} & =\mathbb{E}_{s \in S}\left[\left(\mathcal{F}_{j}(s)-\boldsymbol{\mu}_{j}\right)\left(\mathcal{F}_{j^{\prime}}(s)-\boldsymbol{\mu}_{j^{\prime}}\right)^{T}\right] \\
\boldsymbol{\mu}_{j} & =\mathbb{E}_{s \in S}\left[\mathcal{F}_{j}(s)\right]
\end{aligned}
$$

For stability, we add $\frac{\tau}{d_{j}} \sum_{n=1}^{d_{j}} \operatorname{diag}\left(\boldsymbol{\Sigma}_{j, j}\right)_{n}$ to

\begin{tabular}{|c|c|c|c|c|}
\hline & \multicolumn{4}{|c|}{ loss function } \\
\hline & MSE & MAE & KLD & $(1-\mathrm{COS})^{2}$ \\
\hline \multirow{3}{*}{ 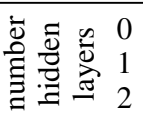 } & $83.0 / 84.2$ & $84.2 / 85.1$ & $83.0 / 84.2$ & $82.4 / 83.5$ \\
\hline & $82.7 / 83.9$ & $83.8 / 84.6$ & 85.1/85.5 & $83.3 / 83.4$ \\
\hline & $82.5 / 82.8$ & $81.3 / 82.1$ & $83.3 / 83.4$ & $82.3 / 82.3$ \\
\hline$\tau=10^{-2}$ & $\tau=10^{-1}$ & $\tau=10^{0}$ & $\tau=10^{1}$ & $\tau=10^{2}$ \\
\hline $84.2 / 84.1$ & $84.8 / 84.7$ & $85.5 / 85.7$ & $85.5 / 86.1$ & $84.9 / 85.9$ \\
\hline
\end{tabular}
$\operatorname{diag}\left(\boldsymbol{\Sigma}_{j, j}\right)$, where $\tau$ is a hyperparameter. We stack the eigenvectors of the top- $d$ eigenvalues into matrices $\boldsymbol{\Theta}_{j} \in \mathbb{R}^{d \times d_{j}}$ and define the GCCA metaembedding of sentence $s^{\prime}$ as:

$$
\mathcal{F}^{\text {gcca }}\left(s^{\prime}\right)=\sum_{j=1}^{J} \boldsymbol{\Theta}_{j}\left(\mathcal{F}_{j}\left(s^{\prime}\right)-\boldsymbol{\mu}_{j}\right)
$$

$\mathcal{F}^{\text {gcca }}$ corresponds to MV-DASE in Poerner and Schütze (2019).
Table 1: Hyperparameter search on STS Benchmark development set for AE (top) and GCCA (bottom). Pearson's $r \times 100 /$ Spearman's $\rho \times 100$.

\subsection{Autoencoders (AEs)}

Autoencoder meta-embeddings are trained by gradient descent to minimize some cross-embedding reconstruction loss. For example, Bollegala and Bao (2018) train feed-forward networks (FFN) to encode two sets of word embeddings into a shared space, and then reconstruct them such that mean squared error with the original embeddings is minimized. Neill and Bollegala (2018) evaluate different reconstruction loss functions: Mean Squared Error (MSE), Mean Absolute Error (MAE), KLDivergence (KLD) or squared cosine distance (1$\operatorname{COS})^{2}$.

We extend their approach to sentence encoders as follows: Every sentence encoder $\mathcal{F}_{j}$ has a trainable encoder $\mathcal{E}_{j}: \mathbb{R}^{d_{j}} \rightarrow \mathbb{R}^{d}$ and a trainable de$\operatorname{coder} \mathcal{D}_{j}: \mathbb{R}^{d} \rightarrow \mathbb{R}^{d_{j}}$, where $d$ is a hyperparameter. Our training objective is to reconstruct every embedding $\mathbf{x}_{j^{\prime}}$ from every $\mathcal{E}_{j}\left(\mathbf{x}_{j}\right)$. This results in $J^{2}$ loss terms, which are jointly optimized:

$$
L\left(\mathbf{x}_{1} \ldots \mathbf{x}_{J}\right)=\sum_{j} \sum_{j^{\prime}} l\left(\mathbf{x}_{j^{\prime}}, \mathcal{D}_{j^{\prime}}\left(\mathcal{E}_{j}\left(\mathbf{x}_{j}\right)\right)\right)
$$

where $l$ is one of the reconstruction loss functions listed above. The autoencoder meta-embedding of a new sentence $s^{\prime}$ is:

$$
\mathcal{F}^{\mathrm{ae}}\left(s^{\prime}\right)=\sum_{j} \mathcal{E}_{j}\left(\mathcal{F}_{j}\left(s^{\prime}\right)\right)
$$

\section{Experiments}

\subsection{Data}

We train on all sentences of length $<60$ from the first file (news.en-00001-of-00100) of the tokenized, lowercased Billion Word Corpus (BWC) (Chelba et al., 2014) ( $\sim 302 \mathrm{~K}$ sentences). We evaluate on STS12 - STS16 (Agirre et al., 2016) and the unsupervised STS Benchmark test set (Cer et al., 


\begin{tabular}{|c|c|c|c|c|c|c|c|c|c|}
\hline \multicolumn{4}{|c|}{ dimensionality } & STS12 & STS13 & STS14 & STS15 & STS16 & STS-B \\
\hline \multirow{3}{*}{\multicolumn{3}{|c|}{$\begin{array}{l}\text { single:ParaNMT } \\
\text { single:USE } \\
\text { single:SBERT }\end{array}$}} & $d=600$ & $67.5 / 66.3$ & $62.7 / 62.8$ & $77.3 / 74.9$ & $\underline{80.3 / 80.8}$ & $78.3 / 79.1$ & $79.8 / 78.9$ \\
\hline & & & $d=512$ & $\overline{62.6} / 63.8$ & $57.3 / 57.8$ & $\overline{69.5 / 66.0}$ & $\overline{74.8} / \overline{77.1}$ & $\overline{73.7} \overline{/ 76.4}$ & $\overline{76.2} / 74.6$ \\
\hline & & & $d=1024$ & $66.9 / \underline{66.8}$ & $\underline{63.2} / \underline{64.8}$ & $74.2 / 74.3$ & $77.3 / 78.3$ & $72.8 / 75.7$ & $76.2 / 79.2$ \\
\hline \multirow{2}{*}{\multicolumn{3}{|c|}{$\begin{array}{l}\text { single:ParaNMT - up-projection* } \\
\text { single:USE - up-projection* }\end{array}$}} & $d=1024$ & 67.3/66.2 & $62.1 / 62.4$ & $77.1 / 74.7$ & $79.7 / 80.2$ & 77.9/78.7 & $79.5 / 78.6$ \\
\hline & & & $d=1024$ & $62.4 / 63.7$ & $57.0 / 57.5$ & $69.4 / 65.9$ & $74.7 / 77.1$ & $73.6 / 76.3$ & $76.0 / 74.5$ \\
\hline \multirow{3}{*}{\multicolumn{3}{|c|}{$\begin{array}{l}\text { meta:conc } \\
\text { meta:avg } \\
\text { meta:svd }\end{array}$}} & $d=2136$ & $72.7 / 71.3$ & $68.4 / 68.6$ & $81.0 / 79.0$ & $84.1 / 85.5$ & 82.0/83.8 & $82.8 / 83.4$ \\
\hline & & & $d=1024$ & $72.5 / 71.2$ & $68.1 / 68.3$ & $80.8 / 78.8$ & $83.7 / 85.1$ & $81.9 / 83.6$ & $82.5 / 83.2$ \\
\hline & & & $d=1024$ & 71.9/70.8 & $68.3 / 68.3$ & $80.6 / 78.6$ & $83.8 / 85.1$ & $81.6 / 83.6$ & $83.4 / 83.8$ \\
\hline \multirow{2}{*}{$\begin{array}{l}\text { meta:gcca } \\
\text { meta:ae }\end{array}$} & (hyperparams & lev set) & $d=1024$ & 72.8/71.6 & $69.6 / 69.4$ & $81.7 / 79.5$ & $84.2 / 85.5$ & $81.3 / 83.3$ & 83.9/84.4 \\
\hline & (hyperparams & dev set) & $d=1024$ & 71.5/70.6 & $68.5 / 68.4$ & $80.1 / 78.5$ & $82.5 / 83.1$ & $80.4 / 81.9$ & $82.1 / 83.3$ \\
\hline \multicolumn{3}{|c|}{ Ethayarajh (2018) } & upervised) & 68.3/- & $66.1 /-$ & $78.4 /-$ & $79.0 /-$ & $-/-$ & $79.5 /-$ \\
\hline \multicolumn{2}{|c|}{ Wieting and Gimpel (2018) } & (un & upervised) & $68.0 /-$ & $62.8 /-$ & $77.5 /-$ & $80.3 /-$ & $78.3 /-$ & 79.9/- \\
\hline \multicolumn{2}{|c|}{ Tang and de Sa (2019) } & (unsuper & ised meta) & $64.0 /-$ & $61.7 /-$ & $73.7 /-$ & $77.2 /-$ & $76.7 /-$ & - \\
\hline \multirow{2}{*}{\multicolumn{2}{|c|}{$\begin{array}{l}\text { Hassan et al. (2019) } \\
\text { Poerner and Schütze (2019) }\end{array}$}} & (unsuper & ised meta) & $67.7 /-$ & 64.6/- & $75.6 /-$ & $80.3 /-$ & 79.3/- & $77.7 /-$ \\
\hline & & (unsuper & jised meta) & $-/-$ & $-/-$ & $-/-$ & $-/-$ & $-/-$ & $80.4 /-$ \\
\hline \multirow{2}{*}{\multicolumn{4}{|c|}{ Reimers and Gurevych (2019) (sup. siamese SoTA) }} & $-/-$ & $-/-$ & $-1-$ & $-/-$ & $-/-$ & $-/ 86.2$ \\
\hline \multicolumn{2}{|c|}{ Raffel et al. (2019) } & & & $-/-$ & $-/-$ & $-/-$ & $-/-$ & $-/-$ & $93.1 / 92.8$ \\
\hline
\end{tabular}

Table 2: Results on STS12-16 and STS Benchmark test set. STS12-16: mean Pearson's $r \times 100 /$ Spearman's $\rho \times 100$. STS Benchmark: overall Pearson's $r \times 100 /$ Spearman's $\rho \times 100$. Evaluated by SentEval (Conneau and Kiela, 2018). Boldface: best in column (except supervised). Underlined: best single-source method. ${ }^{*}$ Results for up-projections are averaged over 10 random seeds. ${ }^{\dagger}$ Unweighted average computed from Hassan et al. (2019, Table 8). There is no supervised SoTA on STS12-16, as they are unsupervised benchmarks.

2017). ${ }^{2}$ These datasets consist of triples $\left(s_{1}, s_{2}, y\right)$, where $s_{1}, s_{2}$ are sentences and $y$ is their ground truth semantic similarity. The task is to predict similarity scores $\hat{y}$ that correlate well with $y$. We predict $\hat{y}=\cos \left(\mathcal{F}\left(s_{1}\right), \mathcal{F}\left(s_{2}\right)\right)$.

\subsection{Metrics}

Previous work on STS differs with respect to (a) the correlation metric and (b) how to aggregate the subtestsets of STS12-16. To maximize comparability, we report both Pearson's $r$ and Spearman's $\rho$. On STS12-16, we aggregate by a non-weighted average, which diverges from the original shared tasks (Agirre et al., 2016) but ensures comparability with more recent baselines (Wieting and Gimpel, 2018; Ethayarajh, 2018). Results for individual STS1216 sub-testsets can be found in the Appendix.

\subsection{Ensemble}

We select our ensemble according to the following criteria: Every encoder should have near-SoTA performance on the unsupervised STS benchmark, and the encoders should not be too similar with regards to their training regime. For instance, we do not

\footnotetext{
${ }^{2}$ We use SentEval for evaluation (Conneau and Kiela, 2018). Since original SentEval does not support the unsupervised STS Benchmark, we use a non-standard repository (https: / / github.com/sidak/SentEval). We manually add the missing STS13-SMT subtask.
}

use Ethayarajh (2018), which is a near-SoTA unsupervised method that uses the same word vectors as ParaNMT (see below).

We choose the Universal Sentence Encoder (USE) $^{3}$ (Cer et al., 2018), which is a Transformer trained on skip-thought, conversation response prediction and Natural Language Inference (NLI), Sentence-BERT (SBERT) ${ }^{4}$ (Reimers and Gurevych, 2019), which is a pre-trained BERT transformer finetuned on NLI, and ParaNMT ${ }^{5}$ (Wieting and Gimpel, 2018), which averages word and 3-gram vectors trained on backtranslated similar sentence pairs. To our knowledge, ParaNMT is the current single-source SoTA on the unsupervised STS Benchmark.

\subsection{Hyperparameters}

We set $d=1024$ in all experiments, which corresponds to the size of the biggest single-source embedding (SBERT). The value of $\tau$ (GCCA), as well as the autoencoder depth and loss function are tuned on the STS Benchmark development set (see

\footnotetext{
${ }^{3}$ https://tfhub.dev/google/ universal-sentence-encoder $/ 2$

${ }^{4}$ https://github.com/UKPLab/ sentence-transformers. We use the large-nlimean-tokens model, which was not finetuned on STS.

${ }^{5}$ https://github.com/jwieting/ para-nmt $-50 m$
} 


\begin{tabular}{l|c|ccc}
\hline & $\begin{array}{c}\text { full } \\
\text { ensemble }\end{array}$ & $\begin{array}{c}\text { without } \\
\text { ParaNMT }\end{array}$ & $\begin{array}{c}\text { without } \\
\text { USE }\end{array}$ & $\begin{array}{c}\text { without } \\
\text { SBERT }\end{array}$ \\
\hline meta:svd & $\mathbf{8 5 . 0 / 8 5 . 4}$ & $79.6 / 81.3$ & $79.7 / 81.4$ & $83.7 / 83.5$ \\
meta:gcca & $\mathbf{8 5 . 5 / 8 6 . 1}$ & $84.9 / 84.8$ & $83.8 / 83.8$ & $85.4 / 85.4$ \\
meta:ae & $\mathbf{8 5 . 1 / 8 5 . 5}$ & $76.5 / 80.3$ & $82.5 / 83.5$ & $28.7 / 41.0$ \\
\hline
\end{tabular}

Table 3: Ablation study: Pearson's $r \times 100 /$ Spearman's $\rho \times 100$ on STS Benchmark development set when one encoder is left out.

Table 1). We train the autoencoder for a fixed number of 500 epochs with a batch size of 10,000 . We use the Adam optimizer (Kingma and $\mathrm{Ba}, 2014$ ) with $\beta_{1}=0.9, \beta_{2}=0.999$ and learning rate 0.001 .

\subsection{Baselines}

Our main baselines are our single-source embeddings. Wieting and Kiela (2019) warn that highdimensional sentence representations can have an advantage over low-dimensional ones, i.e., our meta-embeddings might be better than lowerdimensional single-source embeddings due to size alone. To exclude this possibility, we also upproject smaller embeddings by a random $d \times d_{j}$ matrix sampled from:

$$
\mathcal{U}\left(-\frac{1}{\sqrt{d_{j}}}, \frac{1}{\sqrt{d_{j}}}\right)
$$

Since the up-projected sentence embeddings perform slightly worse than their originals (see Table 2 , rows 4-5), we are confident that performance gains by our meta-embeddings are due to content rather than size.

\subsection{Results}

Table 2 shows that even the worst of our metaembeddings consistently outperform their singlesource components. This underlines the overall usefulness of ensembling sentence encoders, irrespective of the method used.

GCCA outperforms the other meta-embeddings on five out of six datasets. We set a new unsupervised SoTA on the unsupervised STS Benchmark test set, reducing the gap with the supervised siamese SoTA of Reimers and Gurevych (2019) from $7 \%$ to $2 \%$ Spearman's $\rho$.

Interestingly, the naive meta-embedding methods (concatenation and averaging) are competitive with SVD and the autoencoder, despite not needing any unsupervised training. In the case of concatenation, this comes at the cost of increased dimensionality, which may be problematic for downstream applications. The naive averaging method by Coates and Bollegala (2018) however does not have this problem, while performing only marginally worse than concatenation.

\subsection{Ablation}

Table 3 shows that all single-source embeddings contribute positively to the meta-embeddings, which supports their hypothesized complementarity. This result also suggests that further improvements may be possible by extending the ensemble.

\subsection{Computational cost}

\subsubsection{Training}

All of our meta-embeddings are fast to train, either because they have closed-form solutions (GCCA and SVD) or because they are lightweight feedforward nets (autoencoder). The underlying sentence encoders are more complex and slow, but since we do not update them, we can apply them to the unlabeled training data once and then reuse the results as needed.

\subsubsection{Inference}

As noted in Section 2.4, cross-sentence attention systems do not scale well to many real-world STStype tasks, as they do not allow individual sentence embeddings to be cached. Like Reimers and Gurevych (2019), our meta-embeddings do not have this problem. This should make them more suitable for tasks like sentence clustering or real-time semantic search.

\section{Conclusion}

Inspired by the success of word meta-embeddings, we have shown how to apply different metaembedding techniques to ensembles of sentence encoders. All sentence meta-embeddings consistently outperform their individual single-source components on the STS Benchmark and the STS12-16 datasets, with a new unsupervised SoTA set by our GCCA meta-embeddings. Because sentence metaembeddings are agnostic to the size and specifics of their ensemble, it should be possible to add new encoders to the ensemble, potentially improving performance further.

Acknowledgments. This work was supported by Siemens AG and by the European Research Council (\# 740516). 


\section{References}

Eneko Agirre, Carmen Banea, Daniel Cer, Mona Diab, Aitor Gonzalez-Agirre, Rada Mihalcea, German Rigau, and Janyce Wiebe. 2016. SemEval-2016 Task 1: Semantic textual similarity, monolingual and cross-lingual evaluation. In International Workshop on Semantic Evaluation, pages 497-511, San Diego, USA.

Sanjeev Arora, Yingyu Liang, and Tengyu Ma. 2017. A simple but tough-to-beat baseline for sentence embeddings. In ICLR, Toulon, France.

Francis R Bach and Michael I Jordan. 2002. Kernel independent component analysis. JMLR, 3:1-48.

Danushka Bollegala and Cong Bao. 2018. Learning word meta-embeddings by autoencoding. In $C O L$ $I N G$, pages 1650-1661, Santa Fe, USA.

Danushka Bollegala, Kohei Hayashi, and Ken-ichi Kawarabayashi. 2018. Think globally, embed locally - locally linear meta-embedding of words. In ICJAI, pages 3970-3976, Stockholm, Sweden.

Samuel R Bowman, Gabor Angeli, Christopher Potts, and Christopher D Manning. 2015. A large annotated corpus for learning natural language inference. In EMNLP, pages 632-642, Lisbon, Portugal.

Daniel Cer, Mona Diab, Eneko Agirre, Inigo LopezGazpio, and Lucia Specia. 2017. SemEval-2017 task 1: Semantic textual similarity multilingual and crosslingual focused evaluation. In International Workshop on Semantic Evaluation, pages 1-14, Vancouver, Canada.

Daniel Cer, Yinfei Yang, Sheng-yi Kong, Nan Hua, Nicole Limtiaco, Rhomni St John, Noah Constant, Mario Guajardo-Cespedes, Steve Yuan, Chris Tar, et al. 2018. Universal Sentence Encoder for English. In EMNLP, pages 169-174, Brussels, Belgium.

Ciprian Chelba, Tomas Mikolov, Mike Schuster, Qi Ge, Thorsten Brants, Phillipp Koehn, and Tony Robinson. 2014. One billion word benchmark for measuring progress in statistical language modeling. In INTERSPEECH, pages 2635-2639, Singapore.

Joshua Coates and Danushka Bollegala. 2018. Frustratingly easy meta-embedding - computing metaembeddings by averaging source word embeddings. In NAACL-HLT, pages 194-198, New Orleans, USA.

Alexis Conneau and Douwe Kiela. 2018. SentEval: An evaluation toolkit for universal sentence representations. In LREC, pages 1699-1704, Miyazaki, Japan.

Alexis Conneau, Douwe Kiela, Holger Schwenk, Loïc Barrault, and Antoine Bordes. 2017. Supervised learning of universal sentence representations from natural language inference data. In $E M N L P$, pages 670-680, Copenhagen, Denmark.
Jacob Devlin, Ming-Wei Chang, Kenton Lee, and Kristina Toutanova. 2019. BERT: Pre-training of deep bidirectional transformers for language understanding. In NAACL, New Orleans, USA.

Kawin Ethayarajh. 2018. Unsupervised random walk sentence embeddings: A strong but simple baseline. In Workshop on Representation Learning for NLP, pages 91-100, Melbourne, Australia.

Basma Hassan, Samir E Abdelrahman, Reem Bahgat, and Ibrahim Farag. 2019. UESTS: An unsupervised ensemble semantic textual similarity method. IEEE Access, 7:85462-85482.

Sepp Hochreiter and Jürgen Schmidhuber. 1997. Long short-term memory. Neural computation, 9(8):1735-1780.

Douwe Kiela, Changhan Wang, and Kyunghyun Cho. 2018. Dynamic meta-embeddings for improved sentence representations. In EMNLP, pages 1466-1477, Brussels, Belgium.

Diederik P Kingma and Jimmy Ba. 2014. Adam: A method for stochastic optimization. arXiv preprint arXiv:1412.6980.

Tomas Mikolov, Ilya Sutskever, Kai Chen, Greg S Corrado, and Jeff Dean. 2013. Distributed representations of words and phrases and their compositionality. In NeurIPS, pages 3111-3119, Lake Tahoe, USA.

James O' Neill and Danushka Bollegala. 2018. Angular-based word meta-embedding learning. arXiv preprint arXiv:1808.04334.

Jeffrey Pennington, Richard Socher, and Christopher Manning. 2014. GloVe: Global vectors for word representation. In EMNLP, pages 1532-1543, Doha, Qatar.

Matthew Peters, Mark Neumann, Mohit Iyyer, Matt Gardner, Christopher Clark, Kenton Lee, and Luke Zettlemoyer. 2018. Deep contextualized word representations. In NAACL-HLT, pages 2227-2237, New Orleans, USA.

Nina Poerner and Hinrich Schütze. 2019. Multiview domain adapted sentence embeddings for lowresource unsupervised duplicate question detection. In EMNLP-IJCNLP, Hong Kong, China.

Colin Raffel, Noam Shazeer, Adam Roberts, Katherine Lee, Sharan Narang, Michael Matena, Yanqi Zhou, Wei Li, and Peter J Liu. 2019. Exploring the limits of transfer learning with a unified text-to-text transformer. arXiv preprint arXiv:1910.10683.

Pushpendre Rastogi, Benjamin Van Durme, and Raman Arora. 2015. Multiview LSA: Representation learning via generalized CCA. In NAACL-HLT, pages 556-566, Denver, USA. 
Nils Reimers and Iryna Gurevych. 2019. Sentencebert: Sentence embeddings using siamese bertnetworks. In EMNLP-IJCNLP, Hong Kong, China.

Shuai Tang and Virginia R de Sa. 2019. Improving sentence representations with multi-view frameworks. In Interpretability and Robustness for Audio, Speech and Language Workshop, Montreal, Canada.

Ashish Vaswani, Noam Shazeer, Niki Parmar, Jakob Uszkoreit, Llion Jones, Aidan N Gomez, Łukasz Kaiser, and Illia Polosukhin. 2017. Attention is all you need. In NeurIPS, pages 5998-6008, Long Beach, USA.

John Wieting and Kevin Gimpel. 2018. ParaNMT50M: Pushing the limits of paraphrastic sentence embeddings with millions of machine translations. In $A C L$, pages 451-462, Melbourne, Australia.

John Wieting and Douwe Kiela. 2019. No training required: Exploring random encoders for sentence classification. In ICLR, New Orleans, USA.

Wenpeng Yin and Hinrich Schütze. 2016. Learning word meta-embeddings. In $A C L$, pages 1351-1360, Berlin, Germany. 
single-source embeddings

meta-embeddings

\begin{tabular}{|c|c|c|c|c|c|c|c|c|}
\hline $\begin{array}{l}\text { method: } \\
\text { dimensionality: }\end{array}$ & $\begin{array}{c}\text { ParaNMT } \\
d=600\end{array}$ & $\begin{array}{c}\text { SBERT } \\
d=1024\end{array}$ & $\begin{array}{c}\text { USE } \\
d=512\end{array}$ & $\begin{array}{c}\text { conc } \\
d=2136\end{array}$ & $\begin{array}{c}\text { avg } \\
d=1024\end{array}$ & $\begin{array}{c}\text { svd } \\
d=1024\end{array}$ & $\begin{array}{c}\text { gcca } \\
d \stackrel{1024}{=}\end{array}$ & $\begin{array}{c}\text { ae } \\
d=1024\end{array}$ \\
\hline \multicolumn{9}{|l|}{ STS12 } \\
\hline $\begin{array}{l}\text { MSRpar } \\
\text { MSRvid } \\
\text { SMTeuroparl } \\
\text { OnWN } \\
\text { SMTnews }\end{array}$ & \begin{tabular}{|l|}
$55.25 / 55.15$ \\
$88.53 / 88.48$ \\
$53.15 / 59.31$ \\
$73.42 / 69.82$ \\
$67.03 / 58.53$
\end{tabular} & $\begin{array}{l}58.11 / 60.42 \\
87.93 / 89.73 \\
59.63 / 62.40 \\
68.08 / 68.51 \\
60.75 / 53.11\end{array}$ & $\begin{array}{l}34.05 / 39.24 \\
89.46 / 90.75 \\
49.00 / 62.08 \\
71.66 / 65.81 \\
68.66 / 61.29\end{array}$ & $\begin{array}{l}60.13 / 60.53 \\
\mathbf{9 1 . 5 1} / 92.16 \\
58.99 / 64.02 \\
77.89 / 73.05 \\
74.85 / 66.53\end{array}$ & $\begin{array}{l}58.90 / 59.71 \\
91.29 / 91.92 \\
\mathbf{6 0 . 1 6} / \mathbf{6 4 . 7 3} \\
77.53 / 73.00 \\
74.54 / 66.88\end{array}$ & $\begin{array}{l}59.56 / 60.24 \\
91.28 / 91.98 \\
57.03 / 62.17 \\
77.80 / 73.12 \\
73.73 / 66.48\end{array}$ & $\begin{array}{l}\mathbf{6 2 . 7 9 / 6 3 . 9 0} \\
91.20 / 92.29 \\
56.40 / 61.23 \\
\mathbf{7 7 . 9 0} / \mathbf{7 3 . 5 0} \\
\mathbf{7 5 . 7 5} / \mathbf{6 7 . 3 1}\end{array}$ & $\begin{array}{l}61.64 / 63.57 \\
90.69 / 91.69 \\
55.13 / 60.14 \\
75.35 / 73.03 \\
74.91 / 64.76\end{array}$ \\
\hline \multicolumn{9}{|l|}{ STS13 } \\
\hline $\begin{array}{l}\text { FNWN } \\
\text { OnWN } \\
\text { SMT } \\
\text { headlines }\end{array}$ & \begin{tabular}{|l|}
$53.01 / 54.44$ \\
$75.62 / 75.80$ \\
$42.54 / 41.13$ \\
$79.52 / 79.83$
\end{tabular} & $\begin{array}{l}57.06 / 57.22 \\
77.54 / 80.00 \\
44.54 / 44.80 \\
73.67 / 77.17\end{array}$ & $\begin{array}{l}48.07 / 49.34 \\
66.64 / 68.10 \\
43.85 / 41.80 \\
70.70 / 71.82\end{array}$ & $\begin{array}{l}\mathbf{6 4 . 1 1} / \mathbf{6 4 . 9 1} \\
80.84 / 81.13 \\
47.46 / 44.89 \\
81.13 / 83.48\end{array}$ & $\begin{array}{l}63.46 / 64.26 \\
80.46 / 80.81 \\
47.87 / 45.04 \\
80.64 / 82.96\end{array}$ & $\begin{array}{l}63.28 / 63.49 \\
79.89 / 80.53 \\
48.59 / 45.58 \\
81.49 / 83.54\end{array}$ & $\begin{array}{l}62.74 / 63.54 \\
\mathbf{8 4 . 0 4 / 8 3 . 6 5} \\
\mathbf{4 9 . 2 0} / \mathbf{4 6 . 0 1} \\
\mathbf{8 2 . 5 8} / \mathbf{8 4 . 3 7}\end{array}$ & $\begin{array}{l}63.99 / 64.61 \\
80.17 / 81.50 \\
48.92 / 45.40 \\
80.78 / 82.13\end{array}$ \\
\hline \multicolumn{9}{|l|}{ STS14 } \\
\hline $\begin{array}{l}\text { OnWN } \\
\text { deft-forum } \\
\text { deft-news } \\
\text { headlines } \\
\text { images } \\
\text { tweet-news }\end{array}$ & \begin{tabular}{|l|}
$82.22 / 83.20$ \\
$60.01 / 59.49$ \\
$77.46 / 72.75$ \\
$78.85 / 76.98$ \\
$86.14 / 83.36$ \\
$79.39 / 73.43$
\end{tabular} & $\begin{array}{l}81.51 / 82.99 \\
57.66 / 60.45 \\
72.62 / 76.80 \\
73.72 / 75.41 \\
84.57 / 79.42 \\
75.12 / 70.80\end{array}$ & $\begin{array}{l}74.61 / 76.01 \\
50.12 / 49.43 \\
68.35 / 63.35 \\
65.88 / 62.34 \\
85.54 / 80.55 \\
72.48 / 64.24\end{array}$ & $\begin{array}{l}85.08 / 85.83 \\
67.57 / 66.84 \\
\mathbf{8 1 . 7 2} / 79.04 \\
79.64 / 79.93 \\
\mathbf{8 9 . 5 2 / 8 5 . 6 8} \\
82.50 / 76.50\end{array}$ & $\begin{array}{l}85.12 / 85.84 \\
67.09 / 66.19 \\
81.60 / 78.98 \\
79.39 / 79.86 \\
89.35 / 85.51 \\
82.12 / 76.13\end{array}$ & $\begin{array}{l}84.23 / 85.17 \\
66.84 / 66.20 \\
80.36 / 78.31 \\
79.85 / 79.59 \\
89.29 / 85.37 \\
\mathbf{8 3 . 1 4} / 77.17\end{array}$ & \begin{tabular}{|l|}
$87.34 / 87.27$ \\
$\mathbf{6 8 . 4 0 / 6 7 . 2 6}$ \\
$81.09 / 79.20$ \\
$\mathbf{8 1 . 6 8 / 8 1 . 5 0}$ \\
$88.83 / 84.83$ \\
$83.09 / 77.04$
\end{tabular} & $\begin{array}{l}84.24 / 85.09 \\
67.22 / 66.82 \\
79.59 / 78.83 \\
80.13 / 79.77 \\
87.64 / 83.42 \\
81.61 / 77.23\end{array}$ \\
\hline \multicolumn{9}{|l|}{ STS15 } \\
\hline $\begin{array}{l}\text { answers-forums } \\
\text { answers-stud. } \\
\text { belief } \\
\text { headlines } \\
\text { images }\end{array}$ & \begin{tabular}{|l|}
$73.54 / 74.50$ \\
$77.06 / 77.87$ \\
$80.28 / 80.25$ \\
$81.92 / 82.28$ \\
$88.60 / 88.87$
\end{tabular} & $\begin{array}{l}64.04 / 62.78 \\
79.12 / 80.14 \\
77.46 / 77.46 \\
78.91 / 81.88 \\
86.76 / 89.02\end{array}$ & \begin{tabular}{l|}
$72.70 / 75.02$ \\
$60.99 / 63.32$ \\
$78.68 / 82.14$ \\
$73.26 / 74.77$ \\
$88.39 / 90.34$
\end{tabular} & $\begin{array}{l}\mathbf{7 9 . 3 3 / 7 9 . 9 1} \\
81.01 / 82.10 \\
86.14 / 87.58 \\
83.20 / 86.03 \\
\mathbf{9 0 . 9 2 / 9 1 . 9 5}\end{array}$ & $\begin{array}{l}78.47 / 79.12 \\
80.15 / 81.45 \\
85.55 / 87.01 \\
83.33 / 86.25 \\
90.86 / 91.92\end{array}$ & $\begin{array}{l}79.15 / 79.69 \\
81.02 / 82.14 \\
85.05 / 86.02 \\
83.48 / 86.02 \\
90.46 / 91.59\end{array}$ & $\begin{array}{l}78.39 / 78.59 \\
80.86 / 82.18 \\
\mathbf{8 6 . 3 8 / 8 7 . 5 8} \\
\mathbf{8 4 . 8 7 / 8 6 . 7 2} \\
90.34 / 91.85\end{array}$ & $\begin{array}{l}\text { 72.65/72.21 } \\
\mathbf{8 3 . 0 3 / 8 3 . 5 6} \\
82.49 / 83.07 \\
84.16 / 85.53 \\
90.26 / 91.35\end{array}$ \\
\hline \multicolumn{9}{|l|}{ STS16 } \\
\hline $\begin{array}{l}\text { answer-answer } \\
\text { headlines } \\
\text { plagiarism } \\
\text { postediting } \\
\text { question-quest. }\end{array}$ & \begin{tabular}{|l|}
$69.71 / 68.96$ \\
$80.47 / 81.90$ \\
$84.49 / 85.62$ \\
$84.53 / 86.34$ \\
$72.37 / 72.73$
\end{tabular} & $\begin{array}{l}63.41 / 66.63 \\
75.23 / 79.33 \\
80.78 / 82.04 \\
81.32 / 85.87 \\
63.38 / 64.72\end{array}$ & $\begin{array}{l}72.52 / 72.72 \\
69.70 / 75.11 \\
74.93 / 77.42 \\
82.81 / 86.49 \\
68.54 / 70.25\end{array}$ & $\begin{array}{l}\mathbf{7 9 . 6 5} / 78.89 \\
80.97 / 84.95 \\
85.86 / 87.17 \\
\mathbf{8 8 . 1 8} / \mathbf{9 0 . 7 6} \\
75.49 / 77.42\end{array}$ & $\begin{array}{l}78.93 / 77.82 \\
80.60 / 84.53 \\
85.88 / 87.25 \\
87.98 / 90.51 \\
\mathbf{7 6 . 0 5} / 77.76\end{array}$ & $\begin{array}{l}79.37 / 79.21 \\
81.36 / 85.14 \\
85.54 / 87.36 \\
87.55 / 90.21 \\
74.08 / 75.93\end{array}$ & $\begin{array}{l}78.70 / 78.50 \\
\mathbf{8 1 . 4 1} / 84.85 \\
\mathbf{8 5 . 9 2 / 8 7 . 7 6} \\
87.01 / 90.24 \\
73.44 / 74.98\end{array}$ & $\begin{array}{l}76.83 / 77.17 \\
80.40 / 83.17 \\
85.01 / 86.14 \\
86.71 / 89.28 \\
73.25 / 73.60\end{array}$ \\
\hline
\end{tabular}

Table 4: Pearson's $r$ / Spearman's $\rho \times 100$ on individual sub-testsets of STS12-STS16. Boldface: best method in row. 